\title{
CONFINEMENT ENERGY OF QUANTUM DOTS AND THE BRUS EQUATION
}

\author{
Harry S.T. ${ }^{*}{ }^{\square}$, Adekanmbi M. A ${ }^{1 凶}$ iD \\ ${ }^{* 1}$ Department of Physics, Faculty of Natural and Applied Sciences, Ignatius Ajuru University of \\ Education, Rumuolumeni, Port-Harcourt, Rivers State, Nigeria
}

DOI: https://doi.org/10.29121/granthaalayah.v8.i11.2020.2451

Article Type: Research Article

Article Citation: Harry S.T., and Adekanmbi M. A. (2020).

CONFINEMENT ENERGY OF QUANTUM DOTS AND THE BRUS EQUATION. International Journal of Research -GRANTHAALAYAH, 8(11), 318-323. https://doi.org/10.29121/granthaa layah.v8.i11.2020.2451

Received Date: 15 November 2020

Accepted Date: 30 November 2020

Keywords:

Confinement Energy

Quantum Dots

Brus Equation

Spherical Potential Well

Schrodinger Equation

Spherical Bessel Differential

Equation

\begin{abstract}
A review of the ground state confinement energy term in the Brus equation for the bandgap energy of a spherically shaped semiconductor quantum dot was made within the framework of effective mass approximation. The Schrodinger wave equation for a spherical nanoparticle in an infinite spherical potential well was solved in spherical polar coordinate system. Physical reasons in contrast to mathematical expediency were considered and solution obtained. The result reveals that the shift in the confinement energy is less than that predicted by the Brus equation as was adopted in most literatures.
\end{abstract}

\section{INTRODUCTION}

Solid state materials, in general are classified either as metals, semiconductors or insulators. As the name implies, semiconductors are materials whose electrical/electronic properties are intermediate between those of metals and insulators. These intermediate properties are determined by their crystal structures, bonding characteristics, electronic energy level, just to mention but a few (Pillai, 2010). The energy band model or structure of materials dictates that unlike metals, gap, often referred to as forbidden energy gap exist between the valence band and the conduction band of semiconductors and insulators at room temperature. Thus, in loose terms, materials with zero energy gap are metals while those with energy gap greater than $3 \mathrm{eV}$ are commonly referred to as insulators (Yu \& Cardona, 2005). Semiconductors are one of the most useful class of materials ever known to man. Despite that they occupy mainly the groups III and IV in the periodic classification of elements, semiconductors also exist in many different chemical compositions with a large variety of crystal structures. Examples include silicon, germanium, gallium arsenide, cadmium selenide, cadmium sulphide, etc (Yu \& Cardona, 2005). 
The versatility and usefulness of a semiconductor lies in its band gap energy which in turn govern its optoelectronic properties. Band gap engineering refers to the process of altering the band gap energy of a semiconductor to meet specific requirement and application. It is distinct from doping which only shifts the femi level (or energy) within the bandgap of a given semiconductor, thereby creating band tail states either close to the valence band (acceptor state) or conduction band (donor state), leading to the formation of either P-type or N-type semiconductor respectively. Common bandgap engineering techniques include the use of heterostructures (Esaki \& Tsu, 1970) and by alloying composition (Fox \& Ispasoiu, 2017). Decreasing the physical size of a semiconductor into the nanometric region can also be regarded as a bandgap engineering technique as it alters the bandgap due to quantum confinement effects. Semiconductors have made in road into our lives as they are the workhorse of modern electronics. They form the foundation of both industrial and consumer electronics. Improvement in theoretical understanding of the physics of semiconductors coupled with advances in crystal growth and circuit fabrication techniques has led to the development of integrated circuits which come in sizes ranging from small scale integration (SSI) to ultra large scale integration (ULSI) depending on the number of components per chip (Gupta, 2014). This process, often referred to as miniaturization is not without an end. When the material or circuit dimension scales down into the nanometric region, quantum effects become prominent leading to confinement of carriers (electrons and holes). Carriers can be confined in one, two, or three dimensions leading to a quantum well, quantum wire or quantum dot respectively (Davies, 2005). Thus, quantum dots are semiconductor nanostructures (nanoparticles) in which carriers are confined in all three dimensions (Brus, 1984). They are formed predominantly by StranskiKrastanow growth mode, a molecular beam epitaxy technique (Pohl, 2013) and by solution or wet chemistry (Brus, 1983), used to realize colloidal or stand alone quantum dots. Quantum dots exist in different shapes depending on the growth and material conditions. These include spherical, lens, pyramidal, cylindrical shapes, etc. (Pohl, 2013).

Carrier confinement increases the band gap energy of a semiconductor and alters the density of state of the bulk semiconductor material (Pohl, 2013). It therefore extends the frontiers of application of the semiconductor. The energy increase in the band gap is referred to as the confinement energy of the quantum dot.

Brus (1984) gave the first theoretical calculation for a spherical semiconductor colloidal (or stand alone) nanocrystal with CdS and CdSe as examples, based on effective mass approximation. The band gap energy according to Brus is

$$
E_{g(q d)}=E_{b u l k}+\frac{h^{2}}{8 R^{2}}\left(\frac{1}{m_{e}^{*}}+\frac{1}{m_{h}^{*}}\right)-\frac{1.8 e^{2}}{4 \pi \varepsilon_{0} \varepsilon_{r} R^{2}}
$$

Where,

$$
\begin{aligned}
& E_{g(q d)} \quad=\text { band gap energy of quantum dot } \\
& E_{\text {bulk }} \quad=\text { band gap energy of bulk semiconductor } \\
& \mathrm{R} \quad=\text { radius of quantum dot } \\
& m_{e}^{*}=\text { effective mass of excited electron } \\
& m_{h}^{*} \quad=\text { effective mass of excited hole } \\
& \mathrm{h} \quad=\text { planck's constant } \\
& \varepsilon_{0}=\text { permittivity of vacuum } \\
& \varepsilon_{r}=\text { relative permittivity }
\end{aligned}
$$

The first term in the right hand side of equation (1) denotes the band gap energy of the bulk semiconductor. The second additive term in the right hand side of equation (1) represents the additional energy due to quantum confinement. It can be thought of as the infinite square-well contribution to the band gap energy. The third subtractive term stands for the exciton's columbic interaction energy. The numerical factor in this term originates 
from calculations of wave function overlap integrals. Thus, according to the brus equation, the confinement energy $E_{c}$ is explicitly stated as (Brus, 1984):

$$
E_{c}=\frac{h^{2}}{8 R^{2}}\left(\frac{1}{m_{e}^{*}}+\frac{1}{m_{h}^{*}}\right)
$$

One of the short comings of the Brus equation is its failure to account for the electron-hole spatial correlation effect (Kayanuma, 1988). However, our focus in this paper is on the confinement energy term. Quantitatively, the effect of quantum confinement on the band gap energy of a semiconductor is chiefly determined by this term. The Brus equation shows that for a spherical shaped semiconductor nanocrystal, the confinement energy is inversely proportional to the square of the radius of the nanocrystal. The goal of this paper is to investigate this relationship and also ascertain the veracity or otherwise of the confinement energy term. The vast applications of quantum dots in technology is emission based. The confinement energy is important because it determines the emission energy as well as the wavelength of the quantum dot.

\section{FORMALISM}

An ideal spherical quantum dot is a spherical shaped semiconductor nano crystal in which excitons are confined in an infinite spherical well (Delerue \& Lannoo, 2004). This corresponds to an impenetrable hard spherical wall. The confining potential is given by

$$
V_{\text {conf. }}(r)= \begin{cases}0, & r \leq a \\ \infty, & \text { otherwise }\end{cases}
$$

Where,

$$
\begin{aligned}
& \mathrm{R}=\text { radius of the confining potential } \\
& \mathrm{a}=\text { radius of the nanocrystal }
\end{aligned}
$$

Following Schrodinger's time-independent wave equation (Davies, 2005):

$$
\frac{-h^{2}}{2 \mu} \nabla^{2} \Psi(x, \mathrm{y}, \mathrm{z})+\mathrm{V}(x, y, z)=\mathrm{E} \Psi(x, y, z)
$$

Where,

$$
\begin{aligned}
& \mu=\text { mass of particle } \\
& \mathrm{E}=\text { energy of particle } \\
& \Psi=\text { wave function associated with particle }
\end{aligned}
$$

Since the potential depends on the radius from a fixed point of a spherical quantum dot, the Laplacian $\nabla^{2}$ of the spherical polar coordinate is independent of the angular part and is given as

$$
\nabla^{2}=\frac{1}{r^{2}} \frac{\partial}{\partial r}\left(r^{2} \frac{\partial}{\partial r}\right)
$$

Putting equation (4) into equation ( 3 ) for $V=0$ :

$$
\frac{-h^{2}}{2 \mu} \frac{1}{r^{2}} \frac{\partial}{\partial r}\left(r^{2} \frac{\partial}{\partial r}\right)=\mathrm{E} \Psi(x, y, z)
$$


The confinement energy depends only on $r$.

Assume $\Psi(x, y, z)=\mathrm{R}(\mathrm{r}) \mathrm{G}(\theta) \mathrm{Q}(\varnothing)$; The radial part of equation (5) by the method of separation of variables yields:

$$
\frac{1}{R} \frac{d}{d r}\left(r^{2} \frac{d R}{d r}\right)+\frac{2 \mu E r^{2}}{h^{2}}=l(l+1)
$$

Where $l=$ orbital angular momentum

Equation (6) is simplified and the result is stated as :

$$
\frac{d^{2} R}{d r^{2}}+\frac{2}{r} \frac{d R}{d r}+\left[k^{2}-\frac{l(l+1)}{r^{2}}\right] R(r)=0
$$

Where $\mathrm{k}=\frac{\sqrt{2 \mu E}}{h}$

Equation (7) is reminiscent of spherical Bessel differential equation. The solutions are the spherical Bessel function of order $l, j_{l}(k r)$ and the spherical Neumann function of order $l, n_{l}(k r)$.

The general solution is :

$$
R_{n, l}(r)=C_{l} j_{l}(k r)+D_{l} n(k r)
$$

Where $C_{l}$ and $D_{l}$ are constants.

Unlike the spherical Neumann function, the behavior of the Bessel function is such that it is finite at the origin (Weber and Arfken, 2003). The finite requirement of the wave function suggest that D must be equal to zero. This reduces equation (9) to:

$R_{n, l}(r)=C_{l} j_{l}(k r)$

Where,

$$
\begin{aligned}
& C_{l}=\text { normalization constant } \\
& R_{n, l}(r)=\text { eigen function }
\end{aligned}
$$

The wave function must varnish at the boundary. However, in the formulation, due to mathematical expediency, one had used a confining potential that is central, which implies that there exist an explicit hard core potential at the centre. But, according to the physical description of the system, no boundary exist at $r=0$, which would introduce a node in the wave function at that point. Rewriting equation (11) in consonance with Dey et al (2012) yields:

$R_{n, l}(d)=C_{l} j_{l}(k d)$

Where,

$\mathrm{d}=$ diameter of the sphere (distance between two directly opposite points on the sphere).

Also, the infinite potential barrier requires that $\mathrm{R}(\mathrm{d})=0$. This translates into

$$
j_{l}(k d)=0
$$

Where kd is a zero of the lth-order spherical Bessel function.

Unfortunately, the zeros are not located at good (sensible) points (like $\mathrm{n}$ or $\mathrm{n} \pi$ ). 
The boundary condition requires that:

$\mathrm{K}=\frac{1}{d} X_{n, l}$

Where $X_{n, l}$ is the nth zero of the lth-order spherical Bessel function.

Putting equation (12) into equation (7) yields:

$\frac{1}{d^{2}} X_{n, l}^{2}=\frac{2 \mu E}{h^{2}}$

Following equation (13), the allowed energies $E_{n}$ become

$E_{n}=\frac{h^{2}}{2 \mu d^{2}} X_{n, l}^{2}$

Putting $\mu=m_{e}^{*}$ into equation (14) yields the electron confinement energy $E_{e n}$ as :

$E_{\text {en }}=\frac{h^{2}}{2 m_{e}^{*} d^{2}} X_{n, l}^{2}$

Where $m_{e}^{*}$ is the effective mass of electron

Similarly, the hole confinement energy $E_{h n}$ is obtained as:

$E_{h n}=\frac{h^{2}}{2 m_{h}^{*} d^{2}} X_{n, l}^{2}$

Where $m_{h}^{*}$ is the effective mass of hole.

Adding equations (15) and (16) gives the total confinement energy (simply confinement energy), $E_{c}$ as:

$E_{c}=\frac{h^{2}}{2 d^{2}}\left(\frac{1}{m_{e}^{*}}+\frac{1}{m_{h}^{*}}\right) X_{n, l}^{2}$

Where,

$$
\mathrm{n}=\text { radial quantum number }
$$

The ground state corresponds to $\mathrm{n}=1$ and $l=0 . X_{1,0}=3.142$ (Abramowitz and Stegun, 1970) which coincides with the well-known constant $\pi$. Therefore equation (19) becomes:

$E_{c}=\frac{\pi^{2} h^{2}}{2 d^{2}}\left(\frac{1}{m_{e}^{*}}+\frac{1}{m_{h}^{*}}\right)$

\section{CONCLUSION}

The confinement energy is inversely proportional to the square of the diameter of the quantum dot, in contrast to the Brus equation which predicts an inverse square relationship in the radius. The confinement energy based on the brus equation is not entirely new. Very few researches are ground breaking and entirely new. A good number of researches today are innovative and the brus equation is no exception. A "bird eye" view of the brus equation reveals that it is nothing but a Schrodinger equation modified to account for the effect of an electron-hole pair (exciton) 
confined to a nanometric spherical shaped semiconductor referred to as quantum dot. It is blind to the varied crystal structures that exist for semiconductors.

The confinement energy obtained in equation (18) is less (about a quarter) than that predicted by the brus equation adopted in some articles and literatures (Chukwuocha \& Onyeaju, 2012; Ikeri, Onyia, \& Vwavware, 2019) . Unlike the brus equation, equation (19) gives values of the confinement energy for all excited states possible.

\section{SOURCES OF FUNDING}

This research received no specific grant from any funding agency in the public, commercial, or not-for-profit sectors.

\section{CONFLICT OF INTEREST}

The author have declared that no competing interests exist.

\section{ACKNOWLEDGMENT}

None.

\section{REFERENCES}

[1] Abramowitz, M., \& Stegun, I. (1970). Handbook of mathematical functions with formulas, Graphs and mathematical tables. National bureau of standards.

[2] Brus, L.E., (1984). Electron-electron and electron-hole interactions in small semiconductor crystallite: The size dependence of the lowest excited electronic state. J. Chem. Phys. 80, 4403. Retrieved on 18th June, 2020 from http://dx.doi.org/10.1063//1.447218.

[3] Chukwuocha, E.O., Onyeaju, M.C., \& Harry, .S.T., (2012) Theoretical Studies on the Effect of Confinement on Quantum Dots using the Brus Equation. World Journal of Condensed Matter Physics, Vol.2(2), 96-100, USA. Retrieved online on 15th June, 2020 from http://dx.doi.org/10.4236/wjcmp.2012.2017.

[4] Chukwuocha, E.O., \& Onyeaju, M.C., (2012). Effect of Quantum Confinement 0n the Wavelength of CdSe, ZnS and GaAS Quantum Dots (QD). International Journal of Scientific \& Technology Research. Vol. 1, No. 7.

[5] Davies, J.H., (2005). The Physics of Low-Dimensional Semiconductors: An introduction (6th reprint ed.). New York: Cambridge University Press.

[6] Delerue, C. \& Lannoo, M., (2004). Nanostructures: Theory and Modelling. India: Springer.

[7] Dey, S., Swargiary, D., Chakraborty, K., Dasgupta, D., Bordoloi, D., Saikia, R., N ..... Choudhury, S. (2012). The Confinement Energy of Quatum Dots. Retrieved on 19th June, 2020 from https://arxiv.org>pdf

[8] Esaki, L., \& Tsu, R., (1970). Superlattice and Negative differential conductivity in semiconductors. IBM Journal of Research and Developments. Retrieved on 15th June, 2020 from ieeexplore.iee.org

[9] Fox, M., \& Ispasoiu, R., (2017). Quantum Wells, Superlattices, and Bandgap Engineering. In: Kasap., \& Capper, P. (eds). Springer Handbook of Electronic and Photonic Materials. Springer Handbook, Springer, Cham.

[10] Gupta, J.B., (2014). Electronic devices and Circuits. India: S.K. Kataria \&Sons.

[11] Ikeri, H.I., Onyia, A.I., \& VwaVware, O.J., (2019). The Dependence of Confinement energy on the size of Quantum dots. International Journal of Scientific Research in Physics and Applied Sciences, Vol.7, issue 2, pp27-33. Retrieved on 19th June 2020 from https://doi.org/10.26438/ijsrpas/v7i2.2730.

[12] Kayanuma, Y., (1988). Quantum Size Effects of Interacting Electrons and Holes in Semiconductor Microcrystals with Spherical shape. Phys. Rev. B 38, 9797 - 9805.

[13] Pillai, S.O., (2010). Solid State Physics (6th ed.). New-Delhi: New age International.

[14] Pohl, U.W., (2013). Epitaxy of semiconductors: Introduction to Physical Principles Berlin: Springer.

[15] Weber, H.J., \& Arfken, G.B., (2003). Essential Mathematical methods for Physicist. Academic press, USA.

[16] Yu, P.Y. \& Cardona, M., (2005). Fundamentals of Semiconductors: Physics and Material Properties (3rd). New York: Springer. 